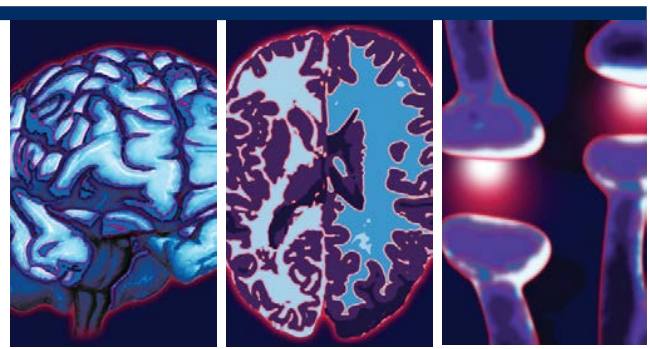

\title{
Time-Interval Estimation Training Modulate Motor Behavior and Cerebral Cortex Activity in Parkinson Disease Patients: Preliminary Study
}

Francisco Magalhães ${ }^{1,2, t}$, Victor Marinho ${ }^{1,2,3, \dagger}$, Carla Ayre ${ }^{1}$, Kaline Rocha ${ }^{1,2}$, Silmar Teixeira ${ }^{1,2}$, Daya Gupta ${ }^{4}$, Victor Hugo Bastos ${ }^{5}$, Mauricio Cagy ${ }^{6}$, Bruna Velasques ${ }^{6}$, Pedro Ribeiro ${ }^{6}$

\begin{abstract}
Background and objectives

Several studies indicate that subjects with Parkinson's disease present motor impaired, timing and many interventions used to improve their motor behavior, but so far no training protocols that use time-estimation tasks. In this preliminary study, we aimed to report the effects of the time-estimation task training on motor behaviour and the electroencephalographic activity of the dorsolateral prefrontal cortex and motor cortex.
\end{abstract}

\section{Methods}

We analysed motor-exploration behaviour in 5 Parkinson's patients using the Unified Parkinson's Disease Rating Scale, in addition to the alpha band absolute power activity of the electroencephalogram.

\section{Results}

Our results show the motor-exploration behaviour improvement in Parkinson patients after the training $(p<0.05)$. Moreover, the alpha band oscillations in the right dorsolateral prefrontal cortex and the motor area bilaterally increased with training $(p<0.05)$.

\section{Conclusion}

We propose that the increase in alpha band absolute power following the training may underlie an efficient accumulation of temporal pulses, which could be responsible for the improvement in the patient motor behaviour demonstrated in the current study.

\section{Keywords}

Time interval, Parkinson's disease, Rehabilitation, Dorsolateral prefrontal cortex, Motor cortex

\section{Introduction \\ Several lines of evidence indicate that Parkinson's disease (PD) subjects present motor impaired timing [1,2]. Therefore, sundry}

rehabilitation interventions used to improve PD subjects' motor behavior, but so far no training protocols that use time-estimation tasks at different time intervals. Teixeira et al.

'NeuroNet, -Neurofeedback Therapie Zentrum-, Parkstrasse 23, D-6606 St. Wendel, Germany

${ }^{2}$ Klinik und Poliklinik für Hals-, Nasen- und Ohrenkrankheiten, plastische und ästhetische Operationen Universität Würzburg Haus B2 Josef-Schneider-Str. 11 D-97080 Würzburg, Germany

${ }^{\dagger}$ Author for correspondences: Francisco Magalhães, Av. São Sebastião no 2819- Nossa Sra. de Fátima- Parnaíba, PI, CEP: 64202-020, Brazil, Tel: +55 86 9993-05639; email: fisiofranciscoxavier@gmail.com

${ }^{\dagger}$ Author for correspondences: Victor Marinho, Av. São Sebastião no 2819- Nossa Sra. de Fátima -Parnaíba, Pl, CEP: 64202-020, Brazil, Tel: +55 86 9417-8117; email: victormarinhophb@hotmail.com 
[3] presented the hypothesis that areas and the brain neuromodulatory mechanisms involved in the time intervals could be used to improve the PD patients' symptoms. They presented the hypothesis based on three attributes: The first refers to the Central Nervous System ability to respond to different time interval oscillations that allow necessary actions to attention and memory. The second was directed to the "internal clock" speed modulation by the dopamine action in the basal ganglia and cerebral cortex. In the third attribute, the authors relate the participation of basal ganglia with automatic movements, cognitive behavior and inhibitory facilitation in the prefrontal and motor cortex [4]. We used electroencephalography in combination with a visual stimulus time estimation paradigm. We hypothesized that time estimation task training over thirty days would increase the alpha band absolute power in the dorsolateral prefrontal cortex (DLPFC) and the motor cortex. Moreover, after training, the patient will present better motor behavior based on the Unified Parkinson's Disease Rating Scale (UPDRS). Thus, this study analyzed the motor behavior and the alpha band absolute power activity in the DLPFC and the PD patients' motor behavior undergo training using a visual time estimation task.

\section{Materials and Methods}

We analysed the cortical changes through the electroencephalography technique (EEG) and motor behavior using the motor domain of the UPDRS scale of five PD patients aged 4070 years (mean $57.8 \pm 7.35$ ) without cognitive impairment. The motor behavior was analyzed pre, middle, and post within thirty days of training with a time estimation task. The task was performed three times a week during one hour of training with regular timetable during the morning. The EEG data was acquired in the first week, fifteen days and at the end of the thirtyday period. In each week, EEG data acquisition occurred at the beginning and end of the week. Of these patients, two had left tremor and one of them had been diagnosed for less than five years. The Parkinson's disease clinical diagnosis was established according to the criteria of the UK Parkinson's Disease Society Brain Bank. There was no history another neurological disease in the Parkinson's disease group. None of the participants had a history of head injury, psychiatric illness or drug/alcohol abuse. Each subject was on medication antiparkinson, taking between 2 and 5 doses per day. During the study, we instructed the patients not to use the medication 24 hours before the start of the training.

EEG recording was done per the protocol used before by Cartier et al. [5]. The time-estimation task consisted of a visual stimulus presentation (yellow circle), in the centre of a 15" monitor randomly in the intervals of $(1,4,7$, and 9s), when the stimulus ceased, the participant estimated how long this stimulus remained on the screen. The software has an additional channel for the EEG that marks the moment the participant started and finished the time estimating task. After the EEG data capture, the alpha band absolute power in the DLPFC and motor area was analyzed according to methods used by Cartier et al. [5].

We used the ANOVA non-parametric (Friedman test) to analyses the motor performance and the alpha band absolute power oscillations in the DLPFC and the motor cortex. The analysis was done at three time-points: before, middle and end of the treatment. We considered data to reach the level of statistical significance if $\mathrm{p} \leq 0.05$. SPSS for Windows version 20.0 (SPSS Inc., Chicago, Il, USA) was used for all analyses.

\section{Results}

The ANOVA non-parametric (Friedman test) for the motor exploration domain of the UPDRS indicated a decrease in the score between pre (Median=12.5), middle (Median=9.00) and post (Median=7.5) treatment, with statistically significant difference, $\left(\chi^{2}(2)=6.857, p=0.03\right)$ (Figure 1A). The alpha band oscillations showed no difference for the left DLPFC $(\mathrm{p}>0.05)$. Statistically significant differences were observed between the pre, middle and posttreatment moments for the left $(\chi 2(5)=60.727$, $\mathrm{p}<0.001)$ (Figure 1B) right motor area $\left(\chi^{2}\right.$ $(5)=59.988, p<0.001$ ) (Figure 1C) and the right DLPFC $(\chi 2 \quad(5)=34.322, \quad \mathrm{p}<0.001)$ (Figure 1D). Post hoc analysis indicated that the alpha band absolute power increased as the participants performed the time-estimation task training $(\mathrm{p}<0.001)$.

\section{Discussion}

This study is the first to seek to understand the effects of time estimation task training on motor behaviour, the DLPFC activity and motor cortex in PD patients. We will discuss our findings 
A

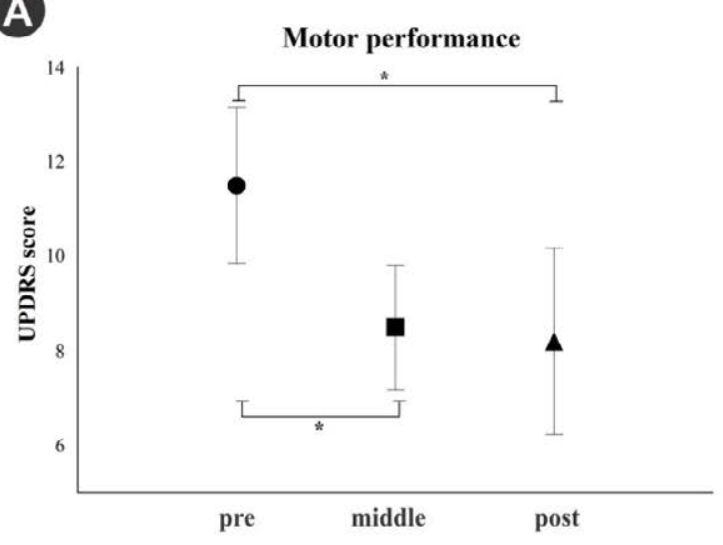

C

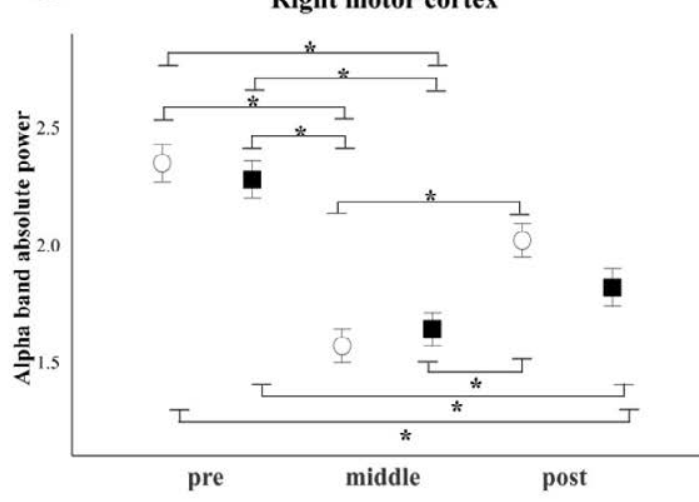

B Left motor cortex

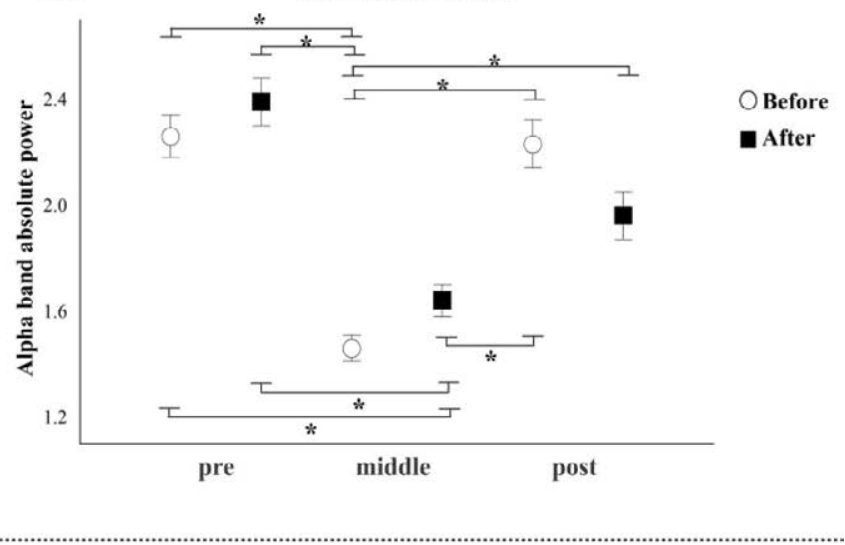

D

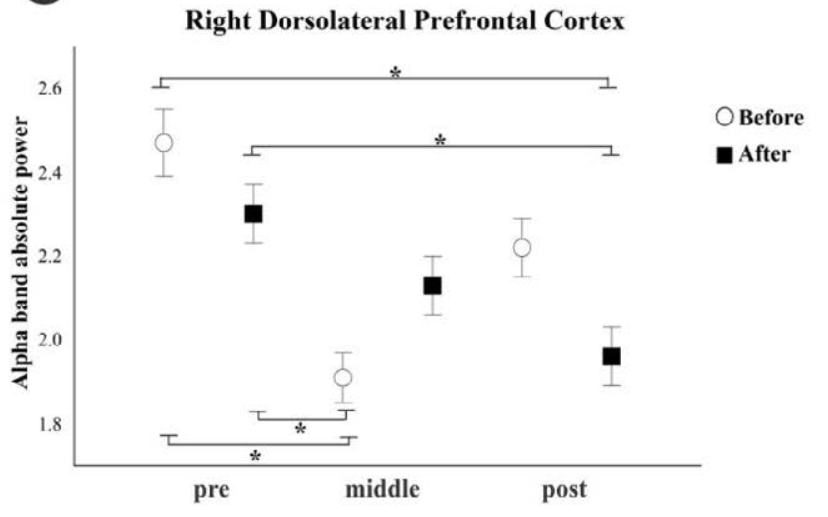

Figure 1: It represents the UPDRS analysis and the alpha band absolute power presented with the average \pm standard deviation in the three moments (pre, middle, and post). Figure 1(A) corresponds to the difference between pre, middle and post for the UPDRS ( $p<0.05$ ), with the lowest value of the UPDRS score corresponding to the best motor performance. Figures $1(B), 1(C)$ and 1 (D) show the difference in alpha power detected at three moments (pre, middle and post-training) for the left motor cortex, right motor cortex and right dorsolateral prefrontal cortex respectively $(*=p<0.05)$, both demonstrated increase power absolute alpha band.

based on the cortical and subcortical structures associating them with the time perception models.

The increase in alpha power over right DLPFC and motor areas on both sides in this study indicates that these regions could be the network part that mediates the timing task effects leading to the improvement of the PD patient's motorexploration behavior [6, 7]. The network structure activated by timing tasks is consistent with multi-synaptic cortical-basal gangliathalamic-cortical loops hypothesized by Gupta [8], which includes the DLPFC in the right hemisphere. This feedback loop is proposed to serve as an oscillator in brain timing mechanisms, which is calibrated by the internal homeostatic demands imposed on the functioning of cortical circuits $[8,9]$. The role of cortical-basal gangliathalamic-cortical loops in homeostatic control of the cortical circuits is consistent with the basal ganglia activation by working memory task [6].

The current study reports the benefits of an explicit perceptual timing task [10], in which subjects estimate the intervals between visual stimuli. The observed gain of explicit timing task on motor exploration behavior of PD patients may be attributed to the plasticity changes in the network for explicit timing function, which includes basal ganglia. Plasticity changes in hubs, common to both working memory and time perception networks, by training, may be responsible for improved calibration of neural temporal units, which would lead to the efficient functional accumulation of pulses (neural temporal units) by internal clocks responsible for timing the motor-exploration behaviour in the current study. Plasticity changes induced by the time-estimation training task may be responsible for the calibration of temporal units, which results in a more accurate representation 
of external physical time in the brain [11]. In a recent genetic phenotype study, in which there is $30-40 \%$ reduction in striatal D2 dopamine receptor density, it was found that the presence of this allele adversely affected the visual interval timing task performance in both sub and suprasecond range [11]. It suggests that changes caused by training in time-estimation task compensate the plasticity changes that may be caused by changes in dopamine release in PD [3]. Thus, training with time-estimation task may help to reduce the dependence on therapeutic drugs in PD patients.

The increase in the alpha power recorded over the right DLPFC and right and left motor areas may indicate changes in the networking of different hubs following timing task training. These changes may help in improved motor-exploration behavior as a result of hubs networking by facilitating the synchronous and asynchronous processing of information [12]. Thus, increased alpha power may represent electrophysiological underpinnings of compensatory changes following training with time-estimation task. Moreover, as it is evident from the above, it will require a multidisciplinary approach to test hypotheses formulated to understand the basis of improvement by time-estimation task training in the PD patients motor exploration behavior.

\section{Conflict of Interest}

Authors declare that the research was conducted in the absence of any commercial or financial relations that could be construed as a potential conflict of interest.

\section{Ethics}

The work described in this article has been carried out by the Ethics Committee of the Federal University of Piauí - UFPI (no 1.087.450) for experiments involving humans; Uniform Requirements for manuscripts submitted to Biomedical Journals.

\section{Acknowledgment}

The acknowledgment of the Brazilian institution that supports science, the National Council for Scientific and Technological Development for encouraging our research.

\section{References}

1. Hove MJ, Keller PE. Impaired movement timing in neurological disorders: rehabilitation and treatment strategies. Ann. N. Y Acad. Sci 1337(1), 111-117 (2015).

2. Shih IF, Liew Z, Krause N, et al. Lifetime occupational and leisure time physical activity and risk of Parkinson's disease. Parkinsonism. Relat. Disord 28(1), 112-117 (2016).

3. Teixeira S, Magalhães F, Marinho V, et al. Proposal for using time estimation training for the treatment of Parkinson's disease. Med. Hypoth 95(1), 58-61 (2016).

4. Jean-Marc M, Curcio G, Mastrolilli F, et al. Alpha and beta EEG power reflects L-dopa acute administration in parkinsonian patients. Front. Aging. Neurosci 6(1), 302
(2014).

5. Cartier C, Diniz C, Di Girogio L, et al. Changes in absolute theta power in bipolar patients during a saccadic attention task. Psychiatry. Res 228(3), 785-790 (2015).

6. Ustun S, Kale EH, Cicek M. Neural Networks for Time Perception and Working Memory. Front. Hum. Neurosci 11(1), 83 (2017).

7. Jones $\mathrm{CR}$, Rosenkranz K, Rothwell JC, et al. The right dorsolateral prefrontal cortex is essential in time reproduction: an investigation with repetitive transcranial magnetic stimulation. Exp. Brain. Res 158(3), 366-372 (2004).

8. Gupta DS. Processing of sub- and supra-second intervals in the primate brain results from the calibration of neuronal oscillators via sensory, motor, and feedback processes.
Front. Psychol 5(1), 816 (2014).

9. Andrea L. Crowell, Elena S, et al. Oscillations in sensorimotor cortex in movement disorders: an electrocorticography study. Brain 135(2), 615-630 (2012).

10. Zelaznik HN, Spencer RM, Ivry RB. Dissociation of explicit and implicit timing in repetitive tapping and drawing movements. J. Exp. Psychol. Hum. Percept. Perform 28(3), 575-588 (2002).

11. Wiener M, Lee YS, Lohoff FW, et al. Individual differences in the morphometry and activation of time perception networks are influenced by dopamine genotype. Neuroimage 89(1), 10-22 (2014).

12. Gupta DS, Chen L. Brain oscillations in perception, timing and action. Curr. Opin. Behav. Sci 8(1), 161-166 (2016). 